January 2010

\title{
A survey comparing the attitudes toward perinatal bereavement care of nurses from three Asian cities
}

\author{
Moon Fai Chan \\ National University of Singapore, Singapore \\ Feng-lan Lou \\ Shandong University, Shandong, China \\ David Arthur \\ Aga Khan University, david.arthur@aku.edu
}

Follow this and additional works at: https://ecommons.aku.edu/pakistan_fhs_son

Part of the Nursing Midwifery Commons

\section{Recommended Citation}

Chan, M., Lou, F., Arthur, D. (2010). A survey comparing the attitudes toward perinatal bereavement care of nurses from three Asian cities. Evaluation and the Health Professions, 33(4), 514-533.

Available at: https://ecommons.aku.edu/pakistan_fhs_son/217 
A Survey

Comparing the

Attitudes Toward

Perinatal

\section{Bereavement Care \\ of Nurses From \\ Three Asian Cities}

(C) The Author(s) 2010

Reprints and permission:

sagepub.com/journalsPermissions.nav DOI: 10.1 I77/0163278710381092

http://ehp.sagepub.com

@SAGE

\author{
Moon Fai Chan', Feng-lan Lou' ${ }^{2}$, and \\ David Gordon Arthur ${ }^{3}$,
}

\begin{abstract}
Caring for parents whose infant has died is extremely demanding, difficult, and stressful. In some situations, nurses may experience personal failure, feel helpless, and need to distance themselves from bereaved parents because they are unable to deal with the enormity of the parental feelings of loss. The aim of the study was to describe and compare attitudes toward perinatal bereavement care across a sample of nurses working in five obstetrics and gynecology settings from three Asian cities, as well as the factors associated with these attitudes. A survey was conducted, and 573 nurses were recruited
\end{abstract}

\footnotetext{
I Alice Lee Centre for Nursing Studies, National University of Singapore, Singapore

${ }^{2}$ School of Nursing, Shandong University, Shandong, China

${ }^{3}$ Angeles University Foundation, Angeles City, Philippines

\section{Corresponding Author:}

Moon Fai Chan, Alice Lee Centre for Nursing Studies, National University of Singapore, Block E3A, Level 3, 7 Engineering Drive I, Singapore II7574, Singapore

Email: nurcmf@nus.edu.sg
} 
from 2006 to 2007. The data were collected using the perinatal bereavement attitudes scale, which involves an II-item self-report questionnaire. Nurses' attitudes were mainly positive, but differed across cities, with the attitude of Jinan nurses being significantly more positive than nurses from the other two cities, and the attitude of Hong Kong nurses being significantly the lowest. Positive attitudes were associated with position, and nurses who were well informed of hospital policy and received training for bereavement care were statistically significantly more likely to have a positive attitude toward perinatal bereavement care. Although nurses' attitudes to prenatal bereavement care differ significantly across the three Asian cities, they are generally similar. The differences observed could be related to the wider social, cultural, and organizational circumstances of nursing practice.

\section{Keywords}

international comparison, perinatal bereavement care, nursing profession

\section{Introduction}

Perinatal loss is defined as stillbirth beyond 24 weeks gestation plus death in the first 28 days of life. Perinatal loss is generally regarded as the most painful form of bereavement because it is unexpected, often sudden and sometimes unexplained (Chambers \& Chan, 2000). The Stillbirth and Neonatal Death Society (SANDS) recommends that all community health practitioners who support bereaved parents should have access to basic, postbasic, and in-service training to equip them to offer adequate care to such families (Stillbirth and Neonatal Death Society [SANDS], 2009). For many bereaved parents, the care that nursing staff provide may have a crucial effect on their response to such a death (Engler \& Lasker, 2000; Rowa-Dewar, 2002). However, caring for and supporting parents whose infant has died is extremely demanding, difficult, and stressful (Gensch \& Midland, 2000; Säflund, Sjögren, \& Wredling, 2004), and in some situations, nursing staff may experience personal failure because they feel helpless and unable to provide help (Robinson, Baker, \& Nackerud, 1999). Although much research has investigated the needs and feelings of bereaved parents in perinatal settings, there has been little mention of the needs and feelings of the perinatal nurses and midwives who care for them in Asian countries. Some nursing staff commented that they need to distance themselves from bereaved parents because they feel unable to deal with the enormity of the parental feelings of loss (Paterson \& Zderad, 1988; Säflund 
et al., 2004). In addition, there has been no research comparing the needs and feelings of perinatal nursing staff across Asian countries.

The theory of culture care diversity and universality was developed by Leininger (1997), who emphasized that perinatal nursing staff should expand their thinking from a unicultural perspective to one that is multicultural, holistic, and comparative. Hence, given the increasing need to work with families of divergent cultures, it is important for these employees to be familiar with the traditions and beliefs of others, as well as what loss means to different individuals (Gibson, 1998). In Asian countries, people treat death as a taboo subject (Ping, Chan, \& Lee, 2002; Yam, Rossiter, $\&$ Cheung, 2001), and attitudes toward bereavement are strongly influenced by the principles of Confucianism and Buddhism, which teach the importance, especially among men, of not crying or expressing negative emotion outside the family (Martinson, 1998; Ping et al., 2002). According to each government statistics, in Singapore, $42.5 \%$ were Buddhism, $15 \%$ are Islam and Christianity, respectively. In China, $25 \%$ were Confucianism and Taoism, 20\% were Buddhism, and $8 \%$ were Christianity; and in Hong Kong, $23 \%$ were Buddhism, 24\% were Christianity, and $11 \%$ were Confucianism. The bereaved person often feels ashamed to show grief, especially outside his or her family (Wu \& Tseng, 1985). However, the pain of losing a young child is revealed in the Chinese saying "The white head witnesses the death of the black head with great pain" (the older generation witnesses the death of the younger generation), and for those with strong traditional beliefs, the pain is compounded by their need to be stoic and not reveal their true feelings at the loss of an infant. For bereaved couples, this often means avoiding any discussion of the death to "protect" relatives such as parents or siblings (Gao, Ting-Toomey, \& Gudykunst, 1996). Since Asian people often avoid open displays of emotion or discussing their feelings, especially with people outside the family, nurses must be able to pick up on the subtle and nonverbal ways of expressing emotion (Ping et al., 2002; Yam et al., 2001). These issues complicate bereavement care and make it difficult for affected parents to seek help. Due to these cultural values or traits, it may not be easy for bereaved parents to express their needs or for perinatal nursing staff to provide adequate help in the bereavement process. The debate about which factors contribute to the formation of certain attitudes becomes even more complex when attitudes are considered from an interregional perspective. Hence, nursing models emphasize the importance of the assessment of bereaved families in a culturally sensitive manner, and they assist caregivers in increasing their effectiveness, overcoming cultural ignorance, and coping better with their own grief responses (Keeney, 
2004; Leininger, 1997). To better understand the nature of attitudes toward perinatal bereavement care, there have been calls for more cross-cultural assessments of the concepts of, beliefs about, and responses to such care. A population studies review by Engler et al. (2004) found that nurses who had more experience and education related to bereavement were more comfortable providing this care. In a Hong Kong study, Yam et al. (2001) found that nurses felt ambivalent and helpless and lacked knowledge and counseling skills in caring for dying infants and their bereaved families. In another study in Hong Kong, Chan, Chan, and Day, (2004) found that nurses' attitudes toward bereavement care were positively correlated with the need for training in bereavement care and support via hospital policies.

Nurses work in a multicultural environment and are expected to understand the needs of Chinese, Malay, and Indian patients; yet, most of the literature is Eurocentric. To help nurses care for and support parents whose baby has died, and cope with their own feelings in this extremely demanding, difficult, and stressful time, it is recommended that special skills are needed to help bereaved parents, and after appropriate training, it is logical to assume that nurses and midwives are better equipped to cope with perinatal bereavement (SANDS, 1991). Typically, nurses receive little education to prepare them for dealing with the death of an infant or for assisting bereaved couples during and after this time (Engler et al., 2004). Szgalsky (1989) and Rybarik (1996) agreed that supportive and educational measures were necessary to help nurses in their work with bereaved individuals.

The aim of the study is to describe and compare attitudes toward perinatal bereavement care held by nurses working in hospital settings across three Asian cities (Hong Kong, Singapore, and Jinan) and the factors associated with these attitudes. The research questions are as follows:

1. What are nurses' attitudes toward perinatal bereavement care?

2. Do nurses' attitudes differ across the three Asian cities?

3. What factors are associated with nurses' attitudes?

\section{Materials and Method}

\section{Settings}

The three cities featured in this study, Hong Kong, Singapore, and Jinan (one of the largest cities in northern China), were part of a universityfunded pilot project. The cities were selected because they represent a cross section of Asian cities with significant commonalities, as well as 
differences in their respective infant mortality statistics. There are, however, notable differences across the cities in the ways that nursing education and bereavement care services are organized. A total of five obstetrics and gynecology (OAG) units from the three cities participated in the study. The average number of beds on each unit was 250, ranging from 220 to 320 .

The power analysis of the study was based on one of the areas of interest, the correlation between nurses' attitudes to training for bereavement care and their attitudes toward perinatal bereavement care. With an expected correlation of .207 (Chan et al., 2004), a minimum of 180 samples was needed from each city for a between-city comparison; this would achieve $80 \%$ power at the 5\% significance level (nQuery Advisor, 2001).

\section{Participants}

Convenience sampling was chosen and involved all nurses working in five OAG units on labor/delivery floors and Neonatal Intensive Care Units (NCIU) in these three cities (one in Singapore, two in Hong Kong, and two in Jinan). The nursing staff who work in the units hold Enrolled or Registered Nurse qualifications and also a midwifery qualification.

\section{Measures}

In 2004, the research team developed an English and Chinese version of a questionnaire to assess the attitudes of perinatal nursing staff toward perinatal bereavement care in Hong Kong (Chan et al., 2004). The questionnaire has two parts; the first is comprised of eight close-ended questions to collect information about nurses' demographic characteristics and experiences related to perinatal care, including age, education level, position, religious belief, personal grieving experiences, past experiences in handling grieving clients, years of work experience in the OAG unit, and any completed training/courses on bereavement care. The second part comprised 11 structured close-ended questions, focusing on nurses' attitudes to perinatal bereavement care ( 4 items), attitudes on the importance of hospital policy to bereavement care ( 3 items), and attitudes concerning the need for bereavement care education/training (4 items; see Table 1). Nurses were asked to rank each item on a 6-point Likert-type scale ranging from (0) Not applicable, (1) Very unimportant or completely disagree, to (5) Very important or completely agree; a high score indicates a positive and favorable attitude. 


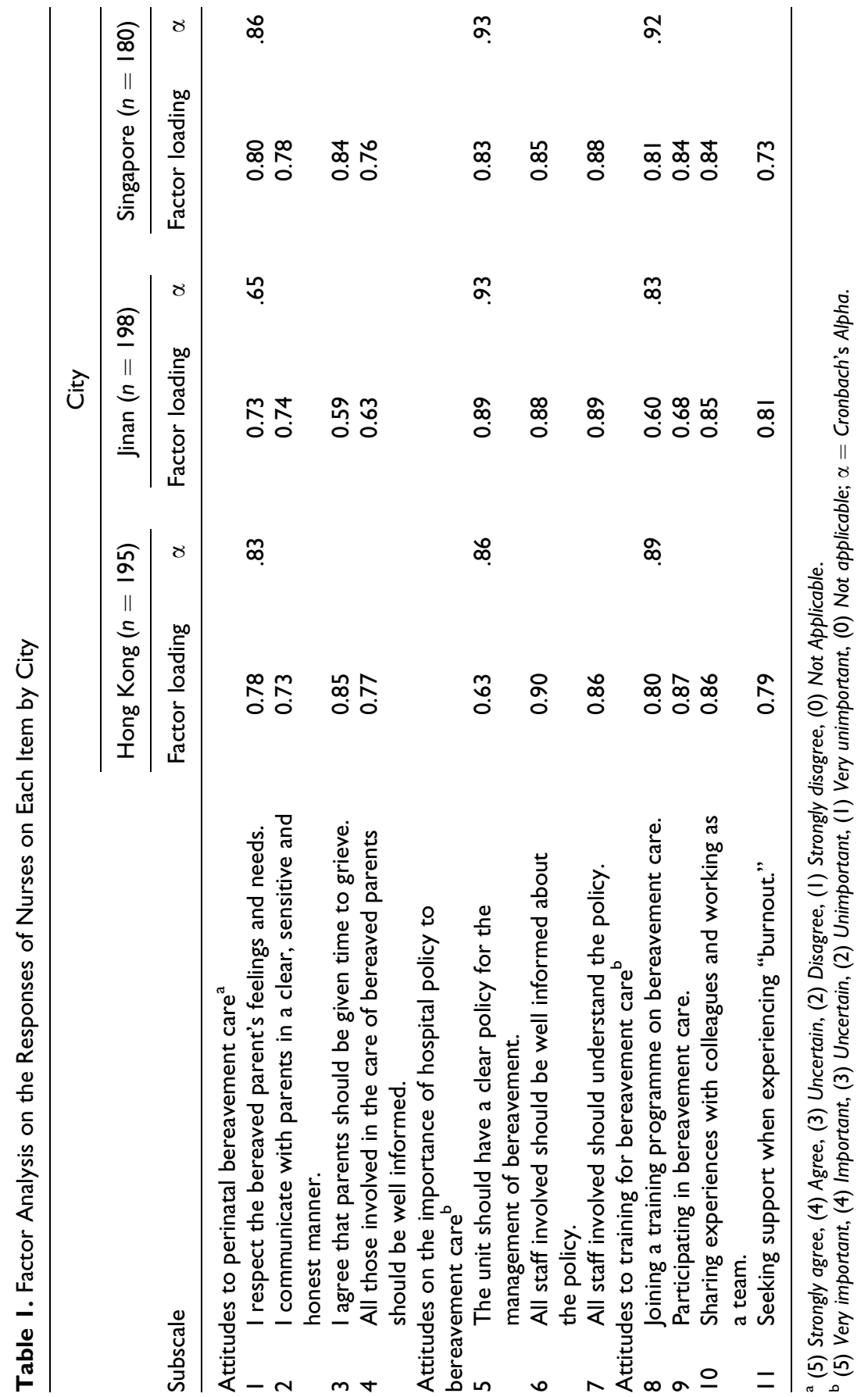




\section{Data Collection}

Data collection took place from 2006 to 2007 using a structured self-report questionnaire. The questionnaire was distributed to nurses at their work place through their ward managers or ward in charge. Instructions were given to the ward manager to first deliver the information sheet to all nursing staff in their ward to explain the aim of the study. The consent form and questionnaire were then sent to each staff. Two boxes were placed in each unit to collect the consent forms and questionnaires separately. Staff IDs and names were not required on the returned questionnaire. Participants were asked to complete and return the consent form and questionnaire within 2 weeks. After 2 weeks, the research team collected the boxes for analysis. These procedures minimized the possibility of ward managers knowing who had participated in the study.

\section{Statistical Analysis}

The data were analyzed using SPSS 16. First, descriptive statistics such as frequencies, percentages, means, and standard errors (SE) were used to describe the study population and average scores of the nurses' attitude. Second, the data were checked for normality, linearity, univariate and multivariate outliers, homogeneity of variance-covariance matrices, and multicollinearity. The distribution of the scores was reasonably normal on all three subscales. Third, a series of one-way multivariate analysis of variance (MANOVA) between groups were performed to investigate whether city and individual characteristics were associated with nurses' attitudes on the three subscales. MANOVA is used because it can test several dependent variables simultaneously (in this case, the three attitude subscales). It controls for the intercorrelations among the various dependent variables, thus protecting against Type I error due to multiple tests of correlated dependent variables (Tabachnick \& Fidell, 2001). Finally, to control for any observed differences in the single variable MANOVAs and explore any confounding influences the variables may have on one another, all variables from the individual tests that showed significance at the $5 \%$ level were entered as covariates into MANOVA, along with the city variable. Where Box's $M$ test of equality of covariance matrices indicated that the assumption of homogeneity of variance-covariance matrices had been violated, the Pillai's trace statistics were used instead of Wilks's lambda to calculate the $F$ value (Polit \& Hungler, 1995). Due to some inequality in sample sizes, the GamesHowell test was chosen for the post hoc comparisons to pinpoint significant 
subscale score differences according to nurses' demographic characteristics and city (Pallant, 2004). For all tests, $p$ values less than .05 were considered statistically significant.

\section{Ethical Approval}

Ethical approval was sought and granted locally in each city. As a principle of sound research, all participants were given an information letter describing the study and explaining that submission of a completed questionnaire would imply consent to participate. It was stressed that participation was entirely voluntary, that all information would be confidentially stored, and that only members of the research team would have access to it.

\section{Results}

\section{Reliability and Validity of the Instrument}

Psychometric properties of the instrument were assessed using principal component factor analysis, Cronbach's $\alpha$ (see Table 1). For the data in Hong Kong, three factors were generated that accounted for $74 \%$ of the variance. Factor 1 accounted for $25.2 \%$ of the variance and reflected concerns related to nurses' attitudes toward perinatal bereavement care. Factor 2 contributed $20.8 \%$ of the variance and focused on the need for bereavement care education/training. Factor 3 comprised items related to hospital policy concerning bereavement care and accounted for $28 \%$. For the data in Jinan, three factors were found that accounted for $67.7 \%$ of the variance, with 19.2, 25.8 , and $22.7 \%$ for Factors 1, 2, and 3, respectively. For the data in Singapore, three factors were found that accounted for $78.3 \%$ of the variance, with 26.3, 24.2, and 27.8\% for Factors 1, 2, and 3, respectively. Cronbach's $\alpha$ was used to examine the internal consistency of the three factors. In Hong Kong, the $\alpha$ values of Factors 1,2 , and 3 were $.83, .86$, and .89, respectively. In the Jinan data, the $\alpha$ values were $.65, .93$, and .83 , respectively, and in the Singapore data, the values were $.86, .93$, and .92 , respectively.

\section{Participant Demographics}

In total, 573 nurses took part in the study: Hong Kong 195 (response rate $86 \%)$, Jinan $(n=198,86 \%)$ and Singapore $(n=180,94 \%)$. There were 70 senior nurses $(12.2 \%)$ and 503 enrolled/registered nurses (87.8\%). More than $30.5 \%(n=175)$ were aged 41 years or older, $39.5 \%(n=226)$ had a religious background and more than $55 \%(n=315)$ had more than 11 years' 
experience in the OAG unit. Only $35.5 \%(n=201)$ held a bachelor's degree or higher in nursing, whereas the remainder were diploma or certificate holders. There were $61.4 \%(n=351)$ with experience in handling grieving clients but less than $21 \%(n=124)$ had taken a bereavement care course. An analysis of the samples' demographic characteristic by city is shown in Table 2.

\section{Comparison of Nurses' Attitudes across the Three Cities}

To test whether the three sub-attitudes of the nurses in the three Asian cities differed, MANOVA was carried out. The results showed that the nurses' attitudes differed across the cities on all three subscales $(p<.001-.042)$. The mean subscale scores of each city are presented in Table 3. First, regarding attitudes toward perinatal bereavement care, nurses practicing in Jinan had more positive attitudes than nurses in the other two cities, whereas Hong Kong nurses had the lowest scores. Second, regarding attitudes toward the importance of formal training to deal with perinatal bereavement care, again, nurses from Jinan had more positive attitudes than nurses from Singapore and Hong Kong and no significant difference was found between Singapore and Hong Kong $(p=.657)$. Finally, with regard to the importance of hospital policy on perinatal bereavement, Singaporean nurses' attitudes were more positive than the other two cities, and again, nurses from Hong Kong had the lowest attitudes scores on this subscale.

\section{Factors Associated With Nurses' Attitudes Toward Perinatal Bereavement Care}

MANOVA was carried out to test whether nurses' personal characteristics had an impact on their attitudes toward perinatal bereavement care. First, eight personal variables were tested separately. Results showed that six of the eight variables were significantly indicative of the differences in nurses' attitudes across all three subscales: age, position, personal grieving experiences, past experiences in handling grieving parents, having taken bereavement care training/courses, and year of experience in the OAG. These results are presented in Table 4 .

In the next step, confounding influences on the observed differences in the single independent variable MANOVAs were controlled for. This was accomplished by entering the six significant variables from these tests into MANOVA as covariates, along with the city variables. The analysis showed that the differences in mean subscale scores, and thus in nurses' attitudes, 
Table 2. Demographic Data of Sample by City

\begin{tabular}{|c|c|c|c|c|}
\hline \multirow[b]{3}{*}{ Variables } & \multirow[b]{3}{*}{ Total $n(\%)$} & \multicolumn{3}{|c|}{ City $(n=573)$} \\
\hline & & $\begin{array}{l}\text { Hong Kong } \\
(n=195)\end{array}$ & $\begin{array}{c}\text { Jinan } \\
(n=198)\end{array}$ & $\begin{array}{l}\text { Singapore } \\
(n=180)\end{array}$ \\
\hline & & $n(\%)$ & $n(\%)$ & $n(\%)$ \\
\hline \multicolumn{5}{|l|}{ Age (years) } \\
\hline $18-25$ & $92(16.1)$ & $34(17.4)$ & 35 (17.7) & $23(12.8)$ \\
\hline $26-30$ & $112(19.5)$ & $39(20.0)$ & $43(21.7)$ & $30(16.7)$ \\
\hline $31-35$ & $65(11.3)$ & $25(12.8)$ & $23(11.6)$ & $17(9.4)$ \\
\hline $36-40$ & $129(22.5)$ & $47(24.1)$ & $44(22.2)$ & $38(21.1)$ \\
\hline $41+$ & $175(30.5)$ & $50(25.6)$ & $53(26.8)$ & $72(40.0)$ \\
\hline \multicolumn{5}{|l|}{ Position } \\
\hline Enrolled/registered nurse & $503(87.8)$ & I $64(84.1)$ & I 78 (89.9) & $161(89.4)$ \\
\hline Senior nurse & $70(12.2)$ & $3 \mid(\mid 5.9)$ & $20(10.1)$ & $19(10.6)$ \\
\hline \multicolumn{5}{|l|}{ Educational level } \\
\hline Diploma or below & $365(64.5)$ & $90(46.9)$ & 142 (7I.7) & $133(75.6)$ \\
\hline Bachelor & $158(27.9)$ & 69 (35.9) & $55(27.8)$ & $34(19.3)$ \\
\hline MSc or above & $43(7.6)$ & $33(17.2)$ & $\mathrm{I}(0.5)$ & $9(5.1)$ \\
\hline \multicolumn{5}{|l|}{$\begin{array}{l}\text { Experience in obstetrics and } \\
\text { gynecology (years) }\end{array}$} \\
\hline$<5$ & $128(22.3)$ & $47(24.1)$ & $34(17.2)$ & $47(26.1)$ \\
\hline $5-10$ & $130(22.7)$ & $50(25.6)$ & $43(21.7)$ & $37(20.6)$ \\
\hline $1 \mathrm{II}$ & $315(55.0)$ & $98(50.3)$ & $12 \mid(6 \mid .1)$ & $96(53.3)$ \\
\hline \multicolumn{5}{|l|}{ Religious belief } \\
\hline Yes & $226(39.5)$ & $85(43.6)$ & $6(3.0)$ & $135(39.5)$ \\
\hline No & $346(60.5)$ & $110(56.4)$ & $192(97.0)$ & $44(24.6)$ \\
\hline \multicolumn{5}{|l|}{ Personal grieving experiences } \\
\hline Yes & $239(42.0)$ & $81(42.0)$ & $45(22.7)$ & $113(63.5)$ \\
\hline No & $330(58.0)$ & $112(58.0)$ & 153 (77.3) & $65(36.5)$ \\
\hline \multicolumn{5}{|c|}{ Taken bereavement training/courses } \\
\hline Yes & $124(21.8)$ & $73(37.8)$ & $7(3.5)$ & $44(24.6)$ \\
\hline No & $446(78.2)$ & $120(62.2)$ & 191 (96.5) & $135(75.4)$ \\
\hline \multicolumn{5}{|l|}{$\begin{array}{l}\text { Past experiences in handling } \\
\text { grieving parents }\end{array}$} \\
\hline Yes & $351(61.4)$ & $130(66.7)$ & $113(57.1)$ & $108(60.3)$ \\
\hline No & $221(38.6)$ & $65(33.3)$ & $85(42.9)$ & 7I (39.7) \\
\hline
\end{tabular}

remained significant only for the position, past experience in handling grieving parents, and city variables (see Table 5). The results of MANOVA indicate that senior nurses had higher scores, and thus more positive attitudes than enrolled/registered nurses, across all three subscales $(p=.003-.01)$. Second, nurses who had past experience in handling grieving parents had higher attitude scores than nurses without such experience across all three subscales $(p<.001-.01)$. Finally, the Games-Howell post hoc test reveals that the differences in nurses' attitude scores according to city all remain 


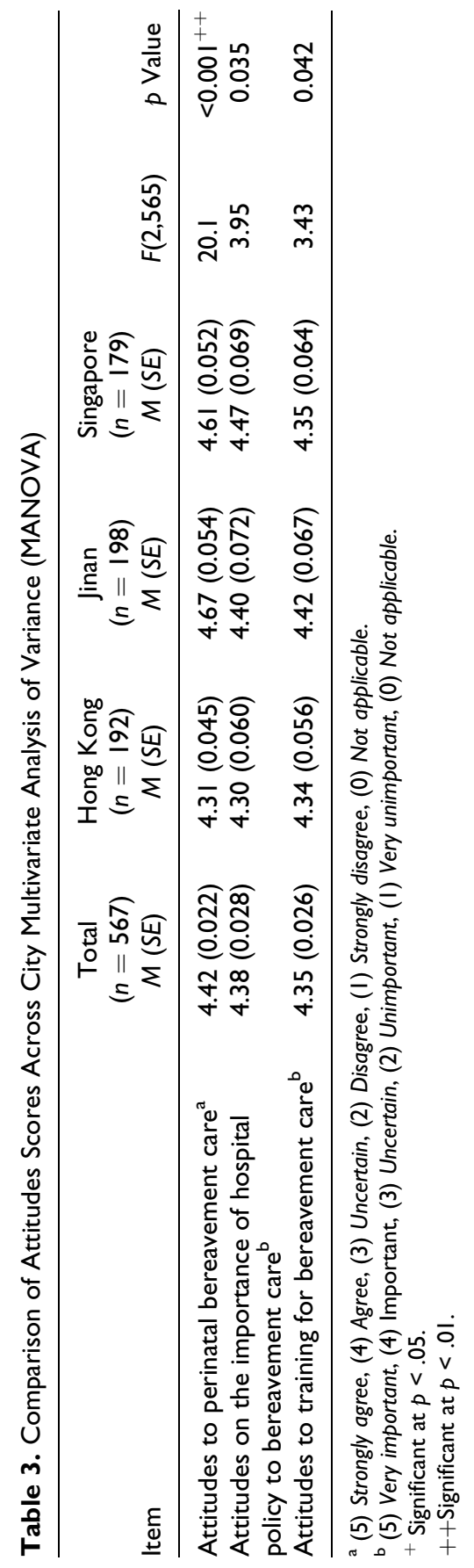




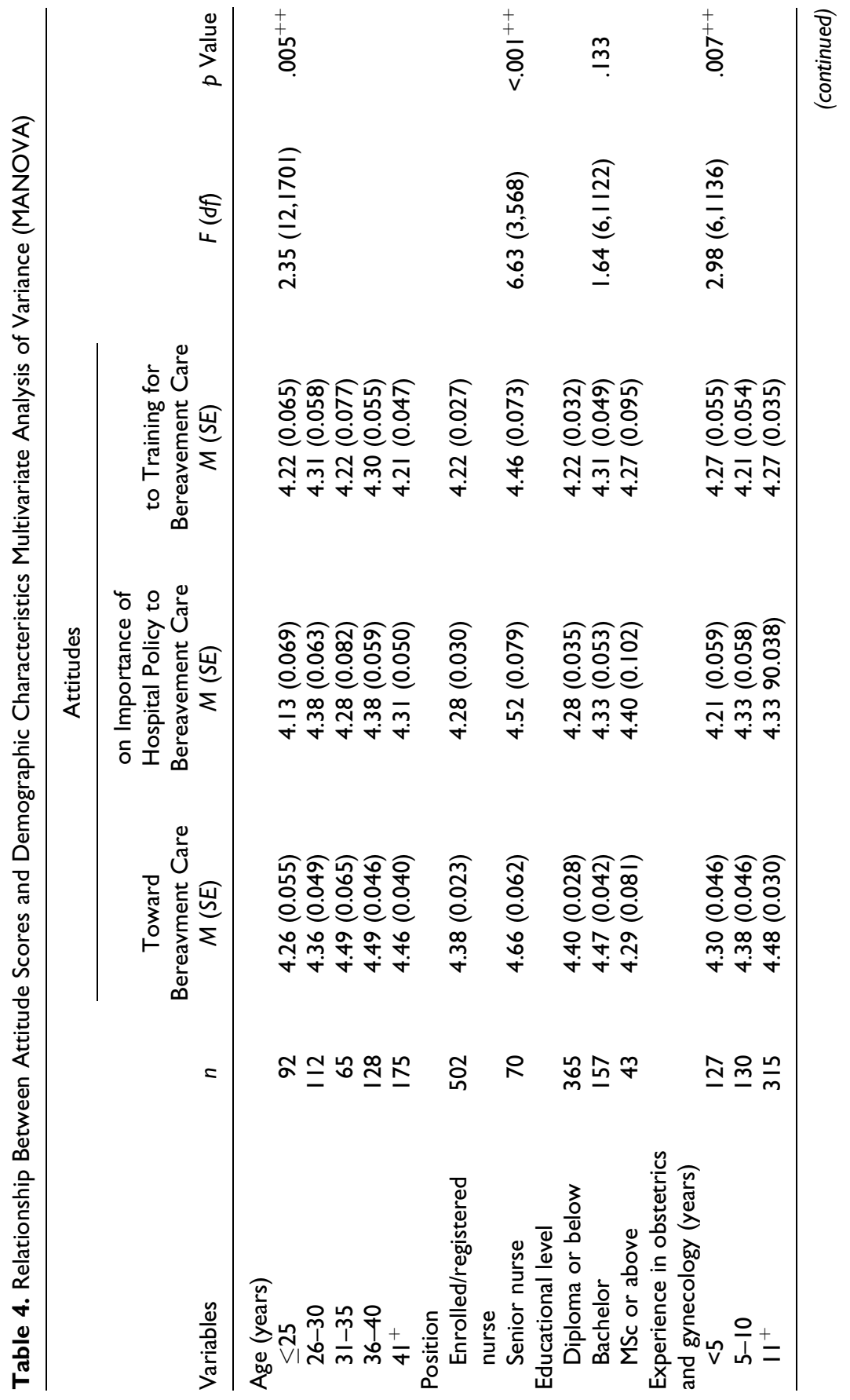




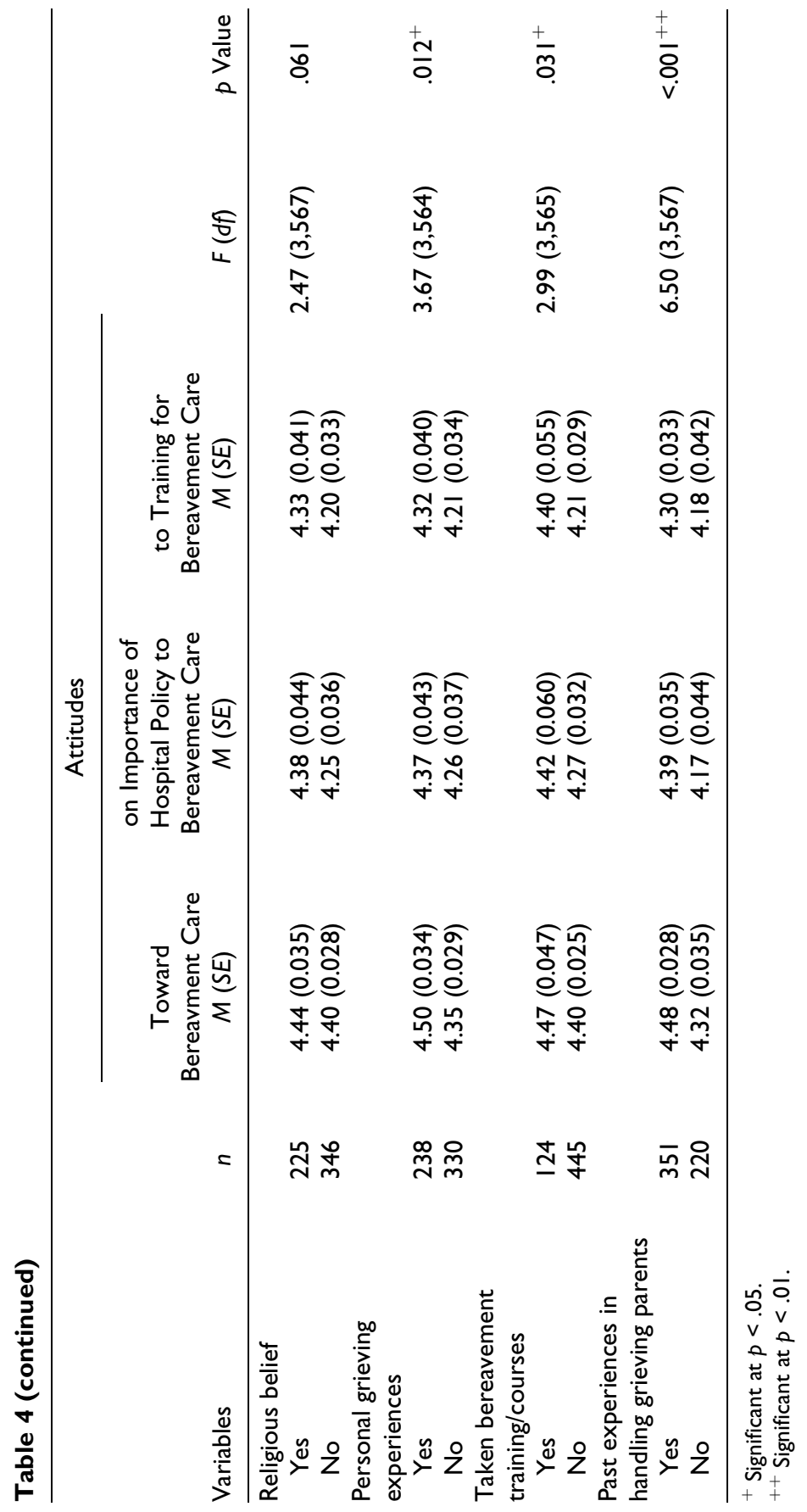




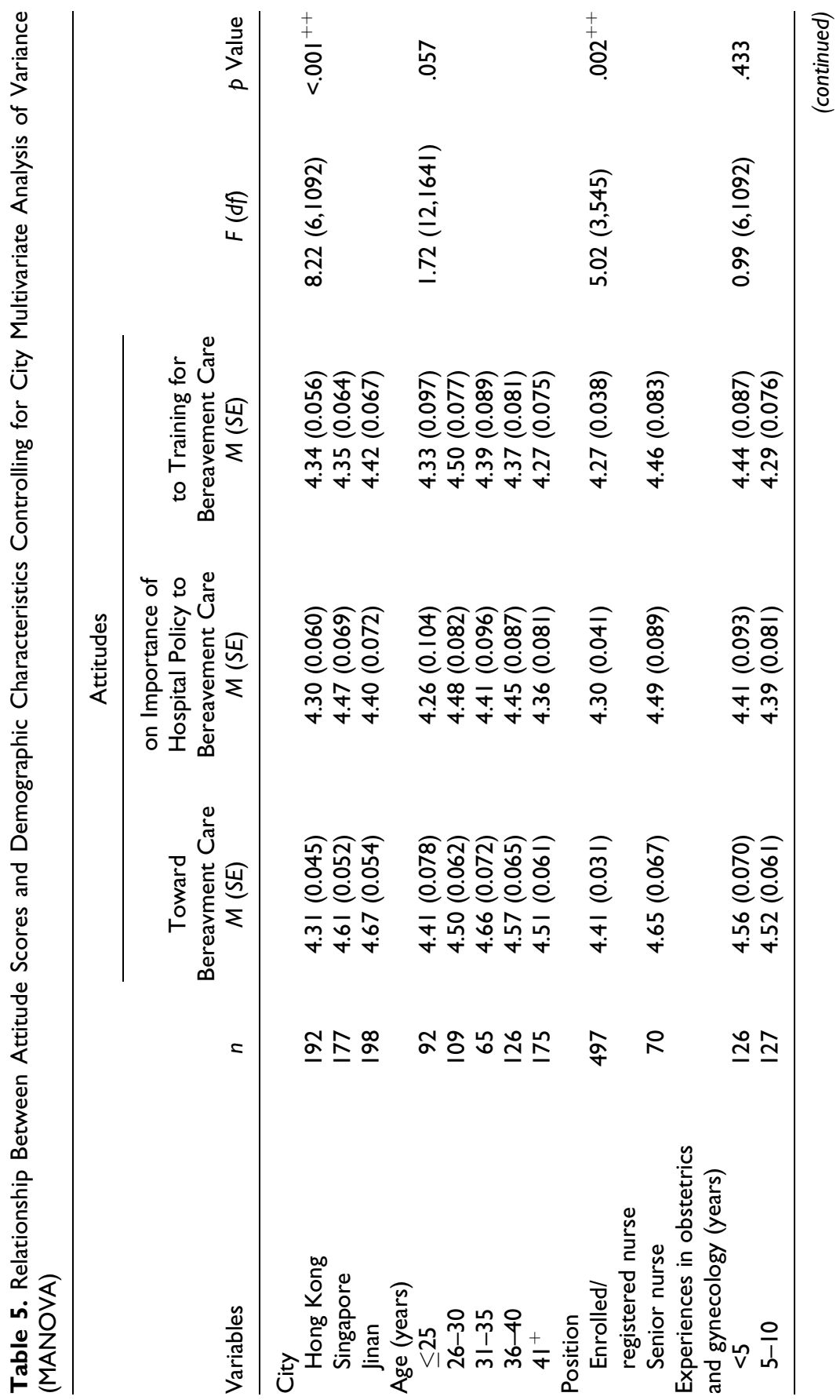




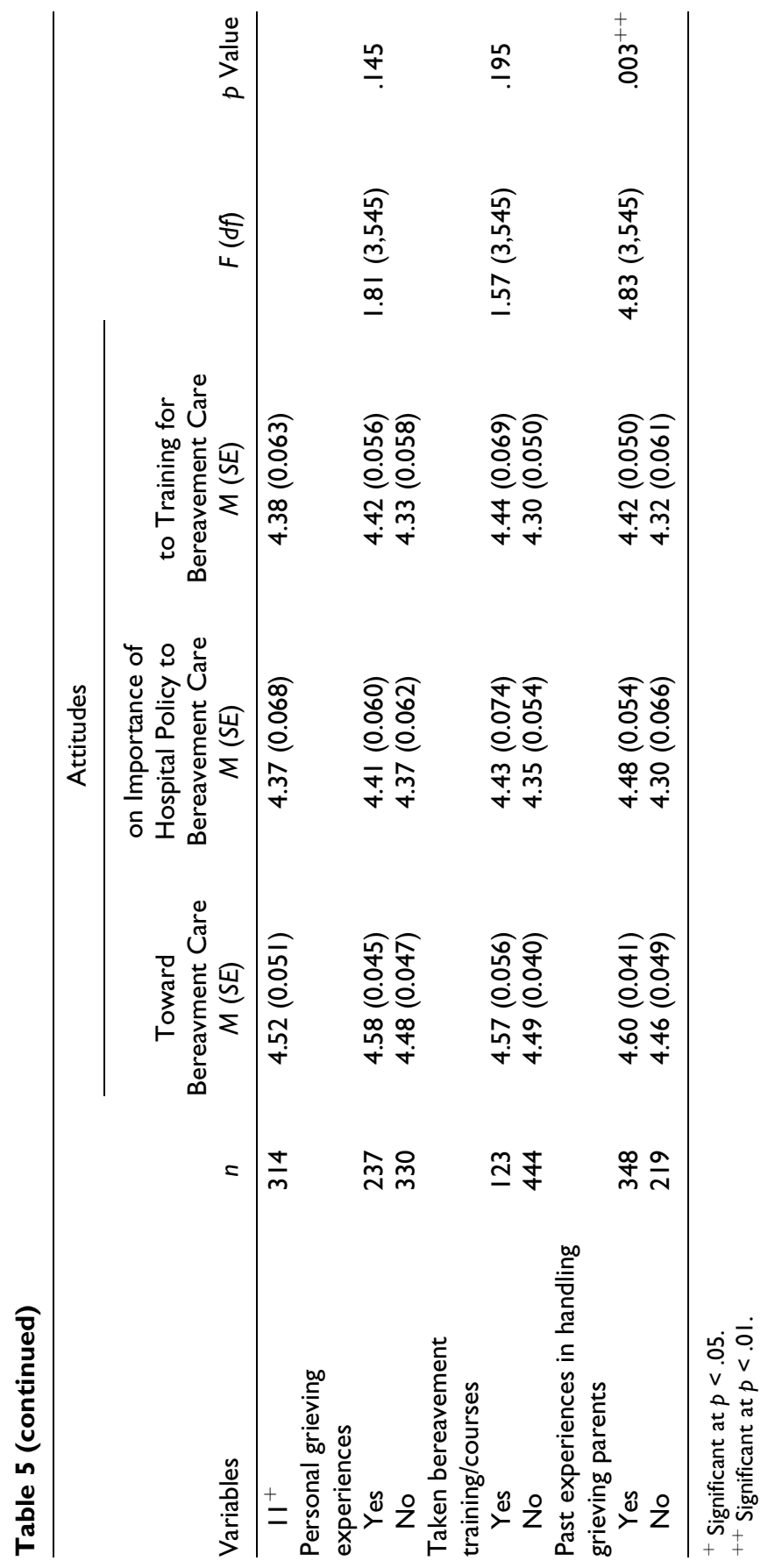


as observed across all subscales ( $p<.01-.02$ ), with the attitudes of Singapore and Jinan nurses more positive than nurses from Hong Kong. Hence, the MANOVA results indicate that the city variable has a confounding effect on the age, experiences in the OAG, personal grieving experiences, and having taken bereavement care training/courses variables, as these variables no longer remain significant when the city variable is added.

\section{Discussion}

The aim of this study was to describe and compare nurses' attitudes toward perinatal bereavement care across three Asian cities and the factors associated with these attitudes.

Significant national differences in nurses' attitudes were also noted by Gardner (1999) and Birtwistle, Paynes, Smith, and Kendrick (2002) and they could in part be explained by the wider organizational and cultural contexts of care. Gardner's study concluded that the main observed differences in attitudes between nurses from Japan, Britain, and the United States, respectively, were primarily due to nationality. She argued that these differences were likely to be part of a general orientation to cultural issues and an extension of the policy surroundings of the organization concerned. These resonate with this study's findings concerning Asian nurses. It may not fully explain national differences, however, as evidenced by the Singaporean nurses seemingly having more positive attitudes toward perinatal bereavement than what was reported in this study. The observed differences could be a function of the culturally dependent ways in which care services, and bereaved parent care services in particular, are organized.

In addition, the aim was to explore which personal characteristics are associated with nurses' attitudes toward bereavement care. Of the eight characteristics surveyed, position and having past experience in handling grieving parents were associated with nurses' attitudes. Senior nurses and one who had past experience in handling grieving parents were found to have a more positive and sympathetic attitude than junior nurses and ones with no experience. Chambers and Chan (2000) suggest that some bereaved parents feel that the care provided by nursing staff made them powerless, and this phenomenon may occur when the focus is on symptoms or parts of the problem rather than on the whole person (Paterson \& Zderad, 1988). Lundqvist, Nilstun, and Dykes (2002) suggested that junior nurses who had no past experience in handling grieving parents treated the bereaved parents as an object. This can convince grieving parents to keep their thoughts to themselves or make them believe that the nursing staff 
were neglecting their thoughts, all of which may lead to insecurity and discouragement. In this study, however, the confounding influences of other personal variables were eliminated. Therefore, the observed position and having past experience differences in the current study are not related to any of the other demographic characteristics that were measured. In contrast to a number of previous studies, the survey results of this study did not find support for the influence on attitudes of age (Engler \& Lasker, 2000), education level (Engler et al., 2004), related bereavement training/courses (Gardner, 1999; Yam et al., 2001), or religious belief (Chan et al., 2004), once the confounding influence of the city variable was added. As mentioned above, it is unclear whether these personal characteristics will affects nurses' attitudes. This is an issue that future research may help clarify. The results also reflect notable cultural differences in the types of nursing qualifications and completed bereavement training/courses across three cities. For example, the data show that the majority of nurses in all cities have not taken any bereavement are training/courses, particular in Jinan, where $96.5 \%$ of the nurses have not. These findings reflect broader organizational differences within the nursing profession, as well as the structural disparities of nurse training across the cities surveyed.

\section{Limitations}

There are some limitations to this regional study. First, due to the small number of nurses surveyed in each city, it is not possible to extend the findings to the general population of nurses within OAG settings in these cities. Second, the homogeneity of the sample introduces systematic bias, as they were from five hospitals in three cities, the majority were Chinese, and their educational level was a diploma or below. Thus, a generalization of the findings to the entire population of interest should be made with caution, and future studies should be based on sufficient sample sizes from more hospitals. Third, the majority of the observed differences in attitude scores, according to personal characteristics, have fairly small effect sizes. The observed differences in attitudes are, therefore, not necessarily noticeable on a practical level. Fourth, a self-report questionnaire was used, which may have caused response bias (Polit \& Hungler, 1995). For example, under some circumstances, socially desirable responses might result in responses appearing more normal or acceptable to the researcher. In addition, as Burns and Grove (2005) have noted, the link between attitudes and actual behavior is tenuous; therefore, this is a weakness of all research concerning attitudes. Finally, given that bereaved parents were not surveyed, no comparisons 
between the perceptions of nurses and parents could be made. Hence, it is recommended that a qualitative study using individual or focus group interviews be carried out to explore the cultural subtleties across the cities and the meaning of perinatal bereavement care for nurses. Despite its methodological limitations, this study appears to represent an important first step in the effort to explore and compare the attitudes toward perinatal bereavement care held by nurses working within Asian health care settings. This is particularly important, as this group of professionals, from a highly demanding area of health care, have traditionally been important as participants of empirical research. Furthermore, this information is of high importance because there is a shortage of nurses and other health care professionals who can work in a cross-cultural city such as Singapore. Where this happens, it will be important to consider cultural influences and perhaps, one solution to address the issue is to liberate the migration of the nursing workforce within Asian countries.

\section{Conclusions}

In general, all of the nurses' attitudes toward perinatal bereavement care are positive. However, nurses' attitudes have been shown to differ across the three cities surveyed. Indeed, cross-cultural research should be undertaken to investigate the presence or otherwise of differences in attitudes in other samples of nurses working in hospital OAG settings across Asian countries. It was discovered that attitudes toward perinatal bereavement care relate to position and past experience in handling grieving parents, irrespective of the nurses' city of practice. Knowing the attitudes of this fundamental group of health care professionals has implications for the educational preparation of all nurses, to ensure that support and education are available to help nurses in their work with bereaved families (Yam et al., 2001). Therefore, the goal of quality bereavement care will be enhanced by addressing the educational and training needs of nurses.

\section{Acknowledgments}

The authors thank for the constructive comments on this article from the editor in chief and the two reviewers.

\section{Declaration of Conflicting Interests}

The author(s) declared no conflicts of interest with respect to the authorship and/or publication of this article. 


\section{Funding}

The author(s) disclosed receipt of the following financial support for the research and/or authorship of this article: Hong Kong Polytechnic University, A-PH28.

\section{References}

Birtwistle, J., Paynes, S., Smith, P., \& Kendrick, T. (2002). The role of the district nurse in bereavement support. Journal of Advanced Nursing, 38, 467-478.

Burns, N., \& Grove, S. K. (2005). The practice of nursing research. Conduct, critique and utilization (5th ed). St. Louis, MO: W. B. Saunders.

Chambers, H. M., \& Chan, F. J. (2000). Support for women/families after peri-natal death. (Cochrane Review), Issue 1. The Cochrane Library, Oxford, Update Software.

Chan, M. F., Chan, S. H., \& Day, M. C. (2004). A pilot study on nurses' attitudes towards perinatal bereavement support: A cluster analysis. Nurse Education Today, 24, 202-210.

Engler, A. J., Cusson, R. M., Brockett, R. T., Cannon-Heinrich, C., Goldberg, M. A., West, M. G., \& Petow, W. (2004). Neonatal staff and advanced practice nurses' perceptions of bereavement/end-of-life care of families of critically ill and/or dying infants. American Journal of Critical Care, 13, 489-498.

Engler, A. J., \& Lasker, J. N. (2000). Predictors of maternal grief in the year after a newborn death. Illness, Crisis \& Loss, 8, 227-243.

Gao, G., Ting-Toomey, S., \& Gudykunst, W. B. (1996). Chinese communication process. In M. H. Bond (Ed.), The handbook of Chinese psychology. Hong Kong: Oxford University Press.

Gardner, J. M. (1999). Perinatal death: Uncovering the needs of midwives and nurses and exploring helpful interventions in the United States, England, and Japan. Journal of Transcultural Nursing, 10, 120-130.

Gensch, B. K., \& Midland, D. (2000). When a baby dies: A standard of care. Illness, Crisis \& Loss, 8, 286-295.

Gibson, M. (1998). Order from chaos. Responding to traumatic events (Rev. ed.). Birmingham, UK: Ventura Press.

Keeney, G. B. (2004). Disaster preparedness: What do we do now? Journal of Midwifery \& Women's Health, 49, 2-6.

Leininger, M. (1997). Overview of the theory of culture care with the ethnonursing research method. Journal of Transcultural Nursing, 8, 32-52.

Lundqvist, A., Nilstun, T., \& Dykes, A. K. (2002). Both empowered and powerless: Mothers' experiences of professional care when their newborn dies. Birth, 29, 192-199. 
Martinson, I. M. (1998). Funeral rituals in Taiwan and Korea. Oncology Nursing Forum, 25, 1756-1760.

nQuery Advisor. (2001). Statistical solutions. Belfast, North Ireland.

Pallant, J. (2004). SPSS survival manual: A step by step guide to data analysis using SPSS for windows (Version 16, 3rd ed). Maidenhead, UK: Open University Press.

Paterson, J. G., \& Zderad, L. T. (1988). Humanistic nursing (2nd ed). New York, NY: National League for Nursing.

Ping, S. L., Chan, Q. H. C., \& Lee, T. F. D. (2002). Use of a bereavement service among suddenly bereaved families in Hong Kong. Journal of Clinical Nursing, 11, 289-290.

Polit, D. F., \& Hungler, B. P. (1995). Nursing research: Principles and methods (5th ed). Philadelphia, PA: Lippincott.

Robinson, M., Baker, L., \& Nackerud, L. (1999). The relationship between attachment theory and perinatal loss. Death Studies, 23, 257-270.

Rowa-Dewar, N. (2002). Do interventions make a difference to bereaved parents? A systematic review of controlled studies. International Journal of Palliative Nursing, 8, 452-457.

Rybarik, F. (1996). What communications skills are most helpful with families grieving a perinatal loss? How can I express my concern while providing appropriate care? AWHONN Voice, 4, 2.

Säflund, K., Sjögren, B., \& Wredling, R. (2004). The role of caregivers after a stillbirth: Views and experiences of parents. Birth, 31, 132-137.

Stillbirth and Neonatal Death Society. (2009). Miscarriage, stillbirth and neonatal death: Guidelines for professionals. London, England: Author.

Szgalsky, J. (1989). Perinatal death, the family, and the role of the health professional. Neonatal Network, 8, 15-19.

Tabachnick, B. G., \& Fidell, L. S. (2001). Using multivariate statistics. New York, NY: Harper Collins.

Wu, D. Y. H., \& Tseng, W. S. (1985). Introduction: The characteristics of Chinese culture. In W. S. Tseng \& D. Y. H. Wu (Eds.), Chinese culture and mental health. Orlando, FL: Academic Press.

Yam, B. M. C., Rossiter, J. C., \& Cheung, K. Y. S. (2001). Caring for dying infants: experiences of neonatal intensive care nurses in Hong Kong. Journal of Clinical Nursing, 10, 651-659. 